ARTICLE

\title{
FLUKA Capabilities and CERN Applications for the Study of Radiation Damage to Electronics at High-Energy Hadron Accelerators
}

Giuseppe BATTISTONI ${ }^{1}$, Vittorio BOCCONE ${ }^{2}$, Francesco BROGGI ${ }^{1}$, Markus BRUGGER ${ }^{2,{ }^{*}}$, Mauro CAMPANELLA ${ }^{1}$, Massimo CARBONI ${ }^{5}$, Francesco CERUTTI ${ }^{2}$, Anton EMPL ${ }^{10}$, Alberto FASSÒ $^{14}$, Alfredo FERRARI ${ }^{2}$, Anna FERRARI ${ }^{15}$, Ettore GADIOLI ${ }^{1}$, Maria Vittoria GARZELLI ${ }^{16}$, Daniel KRAMER ${ }^{2}$, Mattias LANTZ ${ }^{13}$, Elias LEBBOS ${ }^{2}$, Andrea MAIRANI ${ }^{1}$, Annarita MARGIOTTA ${ }^{8}$, Alessio MEREGHETTI' ${ }^{2}$, Cristina MORONE ${ }^{7}$, Silvia MURARO ${ }^{1}$, Katia PARODI ${ }^{9}$, Vincenzo PATERA ${ }^{6}$, Maurizio PELLICCIONI ${ }^{6}$, Lawrence PINSKY ${ }^{10}$, Johannes RANFT ${ }^{4}$, Ketil ROEED ${ }^{2}$, Stefan ROESLER ${ }^{2}$, Sofia ROLLET ${ }^{12}$, Paola R. SALA ${ }^{1}$, Mario SANTANA ${ }^{3}$, Lucia SARCHIAPONE ${ }^{5}$, Massimiliano SIOLI ${ }^{8}$, George SMIRNOV ${ }^{2}$, Florian SOMMERER ${ }^{9}$, Christian THEIS ${ }^{2}$, Stefania TROVATI ${ }^{2}$, Roberto VERSACI ${ }^{2}$, Rosaria VILLARI $^{6}$, Heinz VINCKE ${ }^{2}$, Helmut VINCKE ${ }^{2}$, Vasilis VLACHOUDIS ${ }^{2}$, Joachim VOLLAIRE ${ }^{2}$ and Neil ZAPP ${ }^{11}$

\author{
${ }^{1}$ INFN, Milano, Italy \\ ${ }^{2}$ CERN, 1211 Geneva 23, Switzerland \\ ${ }^{3}$ SLAC, Stanford, U.S.A. \\ ${ }^{4}$ University of Siegen, Siegen, Germany \\ ${ }^{5}$ INFN, Legnaro, Italy \\ ${ }^{6}$ INFN, Frascati, Italy \\ ${ }^{7}$ INFN and University of Roma II, Roma, Italy \\ ${ }^{8}$ INFN and University of Bologna, Bologna, Italy \\ ${ }^{9}$ HIT, Heidelberg, Germany \\ ${ }^{10}$ University of Houston, Houston, U.S.A \\ ${ }^{11}$ NASA, Houston, U.S.A \\ ${ }^{12}$ AIT- Austrian Institute of Technology, Vienna, Austria \\ ${ }^{13}$ Department of Physics and Astronomy, Uppsala University, Uppsala, Sweden \\ ${ }^{14}$ Jefferson Lab, Newport News, U.S.A. \\ ${ }^{15}$ FZD Dresden-Rossendorf, Dresden, Germany \\ ${ }^{16}$ INFN, Italy and University of Granada, Spain
}

\begin{abstract}
The assessment of radiation damage to electronics is a complex process and requires a detailed description of the full particle energy spectra, as well as a clear characterization of the quantities used to predict radiation damage. FLUKA, a multi-purpose particle interaction and transport code, is capable of calculating proton-proton and heavy ion collisions at LHC energies and beyond. It correctly describes the entire hadronic and electromagnetic particle cascade initiated by secondary particles from $\mathrm{TeV}$ energies down to thermal neutrons, and provides direct scoring capabilities essential to estimate in detail the possible risk of radiation damage to electronics. This paper presents the FLUKA capabilities for applications related to radiation damage to electronics, providing benchmarking examples and showing the practical applications of FLUKA at CERN facilities such as CNGS and LHC. Related applications range from the study of device effects, the detailed characterization of the radiation field and radiation monitor calibration, to the input requirements for important mitigation studies including shielding, relocation or other options.
\end{abstract}

KEYWORDS: radiation damage to electronics, CERN, LHC, FLUKA

\section{Introduction}

Modern high-energy hadron accelerators require a significant number of electronic components being placed close to the beam-line and experiments. Recent accelerators require not only superconducting technologies, efficient collimation as well as precise and fast monitoring, but also more complex electronics for control, steering and powering. In many cases cable length or the timing analyzer limits the maximum distance from the equipment to the accelerator, thus it

*Corresponding author, E-mail:Markus.Brugger@cern.ch

(c) 2011 Atomic Energy Society of Japan, All Rights Reserved. leads to equipment being exposed to prompt radiation.

The assessment of radiation damage to electronics is a complex process and requires for the source term a detailed description of the full particle energy spectra, as well as a clear characterization of the calculated quantities used to predict radiation damage. FLUKA, ${ }^{1,2)}$ a multi-purpose particle interaction and transport code, is capable of calculating proton-proton and heavy ion collisions in a wide energy range including LHC (Large Hadron Collider) energies and beyond. It accurately describes the entire hadronic and electromagnetic particle cascade initiated by secondary particles 
from TeV energies down to thermal neutrons, and provides direct scoring functions essential to estimate the possible risk of radiation damage to electronics.

For what concerns radiation effects in electronic devices, ${ }^{3)}$ they can be divided into two main categories: cumulative effects (damage induced by ionizing radiation and displacement of atoms, both resulting in a corresponding slow degradation of microcircuits) and Single Event Effects (SEE). For the former, the steady accumulation of defects causes measurable effects that can ultimately lead to device failure. On the other hand, Single Event Effects are due to the direct ionization by a single particle, able to deposit sufficient energy thereby disturbing normal operation of the device. They can only be characterized in terms of their probability to occur, which will strongly depend on the device as well as on the intensity and kind of the radiation field.

At CERN, ${ }^{4}$ FLUKA is an integral code of all radiation damage studies to electronics. It has been extensively used not only for the experimental areas, but also to address electronics failures at intensive beam loss regions around the LHC accelerator. ${ }^{5)}$ LHC alcoves equipped with commercial or not specifically radiation tolerant electronics are mostly affected by the risk of SEEs, whereas electronics installed in the LHC tunnel will also suffer from accumulated (dose and displacement) damage. For the electronics being installed in these areas the risk of radiation-induced damage has to be minimized as much as possible in order to allow for both safe and efficient LHC operation.

\section{The FLUKA Code and Scoring Capabilities Im- portant for Radiation Damage to Electronics Studies}

\section{Code Overview}

FLUKA $^{1,2)}$ is a general purpose Monte Carlo code capable of handling all radiation kinds from thermal energies (for neutrons), or $1 \mathrm{keV}$ (for all other particles) up to cosmic ray energies and can be applied in many different fields. The validity of the physical models implemented in FLUKA has been benchmarked against a variety of experimental data over a wide energy range, from accelerator data to cosmic ray showers in the Earth atmosphere. ${ }^{1,2)}$

FLUKA is widely used for studies related both to basic research and to applications in particle accelerators. For the latter, FLUKA has proven its huge potential to be used for many applications like target design, beam intercepting devices, windows, dumps, detector optimization, dose estimates, radiological treatment planning, shielding design, as well as studies related to radiation damage. FLUKA is constantly employed in the majority of CERN technical and engineering application.

An integral part of the FLUKA code development is the benchmarking of its physics models and new features against experimental data. It includes both the comparison of predictions of individual models to measurement results (e.g., particle angular distributions and multiplicities) as well as benchmarks in complex situations as, for example, those arising during accelerator operation. Thus, in this paper the applications of the code in terms of radiation damage to electronics as a concern for the LHC will be complemented by application benchmark examples carried out during the operation of the LHC, some of which are mentioned in Section $\mathrm{V}$.

\section{Scoring Capabilities}

As mentioned before, both cumulative as well as stochastic effects lead to possible radiation damage in electronics. For the cumulative part, the user can score dose (particle energy dose) as deposited at any point of interest, as well as a damage equivalent in silicon. The latter probability for a displacement damage caused by non-ionizing energy loss (NIEL) is calculated by scoring the $1 \mathrm{MeV}$ neutron equivalent fluence of all particles in the regions of interest using the implemented displacement damage cross-sections for silicon. ${ }^{6,7)}$ Besides the equivalent fluence, FLUKA also allows to directly score the NIEL and displacement per atom (DPA) as described in the reference. ${ }^{8)}$

The probability of SEEs is estimated by calculating the fluence of hadronic particles with energies greater than a given SEE threshold (usually $20 \mathrm{MeV}$ ) and by then multiplying it with a measured (or estimated) probability of observing a SEE in the device of interest. For this purpose FLUKA allows for a direct scoring of high-energy hadron fluences above $20 \mathrm{MeV}$. The latter threshold is only clearly defined one for charged particles (as they will not get through the housing of the chip at energies below $20 \mathrm{MeV}$ ) and not necessarily true for neutrons. ${ }^{9)}$ Therefore, in the current FLUKA development a new scoring is being implemented and tested including neutrons below $20 \mathrm{MeV}$, in particular for two energy ranges of interest:

- below $20 \mathrm{MeV}$ down to a selectable threshold of a few $\mathrm{MeV}$ and following a fit function corresponding to the respective cross-section decrease

- thermal neutrons up to a few tens of eV following a logarithmic normalization equivalent to typical capture cross sections (e.g., on Boron)

Both scorings allow for a set of user defined parameters (i.e., neutron threshold, fitting function or thermal neutron scaling), in order to adopt the scoring the most appropriate way for the electronic device of interest.

In addition, LET scoring and detailed tracking of fragments is possible if required for device related calculations.

\section{Radiation Damage to Electronics Studies for the LHC}

Radiation levels expected in various LHC underground areas, in particular the integrated flux of high-energy hadrons (multi-MeV range and higher) pose a significant risk to all electronics if they are not specifically designed or tested for such radiation levels. The risk of failure of electronics due to the SEEs previously mentioned becomes high since electronics control logic is present in an increasing number of equipment. Such failures can lead to serious consequences: for instance, SEEs were responsible for the stop 
of the CNGS ("CERN Neutrinos to Gran Sasso”) facility. In this case prompt radiation perturbed the running of so-called PLCs (Programmable Logic Controllers) that controlled the cooling and ventilation system, and led to an undetected failure of the fire detection and problems with the access system. This incident lead to the careful evaluation of all electronic systems installed in all the LHC underground areas. This work, as well as the study and coordination of mitigation options, is performed by a working group on "Radiation to Electronics (R2E)". 10)

The R2E working group includes experts in the various fields related to electronics damage and coordinates all respective activities for the LHC accelerator. It assists LHC operations and equipment groups with assessments of radiation-induced failures in electronics of accelerator components, in order to minimize all risks of radiation-induced failures at CERN accelerators, starting with the LHC.

The analysis process, finally leading to proposed mitigation actions, includes the review of radiation levels (first through detailed Monte Carlo simulations and later by comparison with measurements), the collection of detailed information of the equipments and monitor locations. The simulation data are continuously evaluated and radiation levels are extrapolated for both early operation, and nominal LHC operational conditions. The aim is to provide maximum fluence/dose values for each critical underground area, considering all related uncertainties (assumptions in calculations, equipment sensitivities, operational constraints, etc...). The information is provided to the responsible for the equipment and is to be used as design criteria for the new equipment to be installed in the respective locations.

In the context of reducing the risk of radiation damage to electronics, mitigation actions can be split into three main categories:

\section{Shielding \\ 2. Relocation \\ 3. New Developments}

As relocation cannot be applied to all installed electronics, the detailed study of the radiation field and the design of shielding is an important method to reduce radiation levels in the most critical areas and allow for safe operation of the equipment.

\section{Shielding for Radiation Damage of Electronics at High-Energy Hadron Accelerators}

The cascades originating from beam interactions involve four basic source terms:

- $\quad$ beam/beam interactions (at and close to the experiments),

- beam/residual-gas interactions (all along the accelerator)

- $\quad$ direct losses: beam cleaning at collimator locations or beam dump

- $\quad$ spurious losses (not defined locations).
The emerging secondary particle cascade is then again defining a multi particle-type and energy spectrum, interacting with the various materials around the accelerator.

Therefore, in order to reduce the radiation levels and the corresponding fluences, shielding material can be employed to initiate and absorb showers in order to protect the most sensitive electronics from damage. If the shielding material is thick, the cascades continue until most of the charged particles and photons have been absorbed, except for neutrons and secondary photons.

Shielding design calculations are an important application for FLUKA. The following aspects have to be considered in the conceptual shielding design:

\section{Attenuation of Radiation}

The energy loss of high-energy charged particles is mostly due to ionization. The cross sections for this process are large and accounts for the majority of the energy loss in electromagnetic showers and for about $2 / 3$ of the energy deposited in hadronic showers. Most of the other $1 / 3$ of the hadronic energy is carried away by neutrons. Having no charge these are not involved in ionization loss, are decoupled from the rest of the shower, are subject to elastic and inelastic nuclear collisions and are considerably more penetrating than the charged component.

\section{Shielding of Electromagnetic Showers}

Electromagnetic showers in the $\mathrm{GeV}$ range mainly form through successive bremsstrahlung and pair production. The particles involved are photons, electrons and positrons which have typical radiation lengths in dense materials in the centimeter range. As a result high-energy electromagnetic showers have a characteristic longitudinal dimension of tens of centimeters and are halted by a typical concrete shielding wall having thickness of the order of 1 meter. It has to be noted that secondary neutrons are also produced through photo-production, thus associated shielding issues have to be appropriately considered.

\section{Shielding of Charged Hadrons}

Hadronic showers above $100 \mathrm{MeV}$ progress through a variety of hadronic and nuclear processes that result in secondaries that are predominantly pions $\left(\pi^{+}, \pi^{-}, \pi^{0}\right)$, followed in importance by nucleons (protons, neutrons), strange mesons/baryons and photons. The $\pi^{0}$ component is particularly important as it appears whenever hadron energies are above the single pion production threshold. The $\pi^{0}$ then decays immediately to two photons producing again electromagnetic showers and shifting the shower energy from the hadronic to the electromagnetic sector. Hadrons above few tens of $\mathrm{MeV}$ undergo nuclear reactions that increase the neutron multiplicity. The spallation reactionbreaks the nucleus into a few large fragments. Additional neutrons may also be produced just during this process, or by a subsequent evaporation from these excited fragments. Since the fragments ionize and have large mass (M) and total charge (Z) they will be stopped quickly in dense shield materials of several centimeter thicknesses. 


\section{Shielding of Neutrons}

The neutron elastic scattering cross section on nuclei is large at all energies and which can be employed in shielding applications to attenuate neutrons. The scattered neutron will lose energy on every elastic collision, especially if the target nucleus is light, and the recoils carry away the remaining fraction of the energy. Therefore, hydrogen and light elements are the best in reducing the neutron kinetic energy. Even though neutrons may not be removed during the process of 'moderation', moderation is still effective as in the majority of cases high-energy neutrons are the most damaging to electronics. Moderation can drop their energies below damage thresholds. However, special care must be taken in two cases:

- $\quad$ neutrons streaming through ventilation channels or other penetrations where intermediate and low-energy neutrons readily scatter from the wall. Propagation down the channel is thus possible via a series of 'reflections' even if the channel is not in the original direction of the neutron.

- For thermalized neutrons certain electronic components (especially) might show an increased (or even dominant) failure cross-section due to capture reactions (e.g., the ${ }^{10} \mathrm{~B}\left(\mathrm{n}\right.$,alpha) ${ }^{7} \mathrm{Li}$ reaction) happening close to the sensitive region inside the chip.

With respect to the shielding of electromagnetic showers and charged hadrons, efficient neutron shielding is therefore more difficult to achieve in the tunnel of the LHC and detailed case-by-case Monte Carlo studies are required in order to optimize the shielding design.

\section{Benchmark and Application Examples}

\section{Shielding Penetration Benchmark}

As mentioned in the previous section, the attenuation of high-energy hadrons through thick shielding is essential when one considers to protect electronics installed close to the accelerator. Shielding installations are usually composed of either iron, concrete or combined layers of both materials. Shielding thicknesses can easily reach several meters, thus the benchmark of penetration studies in this radiation environment is very important.

An attenuation benchmark inter-comparison of the predictions of different Monte Carlo codes, including FLUKA, with experimental data obtained at $\mathrm{BNL}^{11)}$ and a benchmark which has been published recently by the SATIF-10 Task Force $^{12)}$ with some results also being presented in this conference. $^{13)}$ The experiment, carried out at the Alternating Gradient Synchrotron (AGS) accelerator in Brookhaven, consisted in activating Bi-foils behind layers of concrete and steel shielding of different thicknesses which were placed laterally to a mercury target. Two proton beam energies were used, $2.83 \mathrm{GeV}$ and $24 \mathrm{GeV}$. The benchmark study showed a very good agreement between calculated and measured reaction rates for the creation of ${ }^{204} \mathrm{Bi}$ and ${ }^{206} \mathrm{Bi}$ nuclides in the

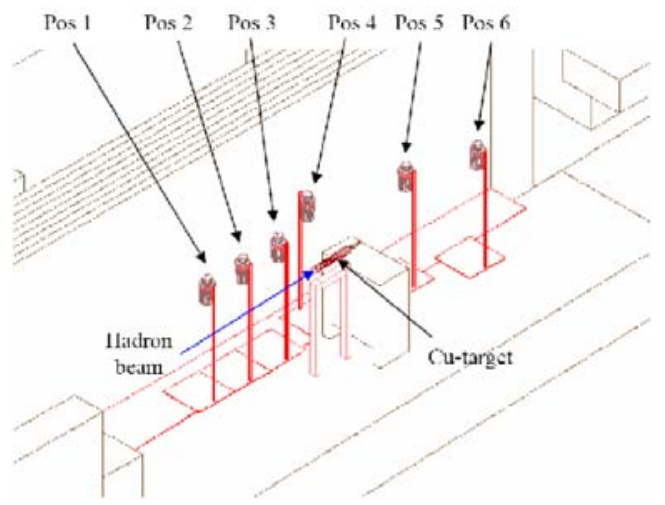

Fig. 1 CERF layout around the copper target indicating the six measurement positions as used for the benchmark measurements

foils as function of depth inside the shielding. This benchmark explores high-energy neutron fluences through the shielding due to the high reaction threshold energies (20$40 \mathrm{MeV}$ ) leading to the production of the bismuth isotopes. This activation benchmark is thus of high relevance for radiation damage studies for electronics where similar thresholds exist. The results demonstrate that FLUKA reproduces the data within $40 \%$ up to several meters of shielding, a very good agreement was obtained for the data taken by the large experimental setup and the important thickness of the shield.

\section{Benchmark Measurements at the CERF Facility}

In order to obtain measurements in a radiation environment similar to the LHC, however still within a well controlled setup, a set of measurements was performed at the CERF benchmark facility. ${ }^{14)}$ A layout of the CERF experimental area is shown in Fig. 1. A pulsed, $120 \mathrm{GeV} / \mathrm{c}$ mixed hadron beam $(60.7 \% \pi+$, 34,8\% p, $4.5 \% \mathrm{~K}+$ ), from the Super Proton Synchrotron (SPS) accelerator, is aimed at a $50 \mathrm{~cm}$ copper target creating a radiation field similar to what can be expected in the beam loss regions in the LHC tunnel. The CERF facility can therefore be used as reference field for calibrating and testing of equipment monitoring damage to electronics in a mixed radiation field. Variation in this radiation field can be achieved by placing the equipment at different positions around the copper target. These positions are indicated by labels ranging from 1 through 6 , where the downstream positions (4-6) are representative for the LHC tunnel regions, whereas the other positions (1-3) are very similar to shielded areas close to the accelerator tunnel.

So-called RadMon detectors ${ }^{15,16)}$ were used for the benchmark at the CERF facility as the RadMon provides online measurements of the Total Ionizing Dose (TID as measured with a RadFET), the $1 \mathrm{MeV}$ neutron equivalent fluence (measured with a PIN-diode ) and the high-energy (> $20 \mathrm{MeV}$ ) hadron fluence (measured by "counting” SEUs). Each RadMon can be operated at $5 \mathrm{~V}$ or $3 \mathrm{~V}$ where the lower voltage effectively increases its Single Event Upset (SEU) sensitivity. During the past years and in view of their high importance for the LHC, a significant effort was made in order to improve their calibration. ${ }^{9)}$ 


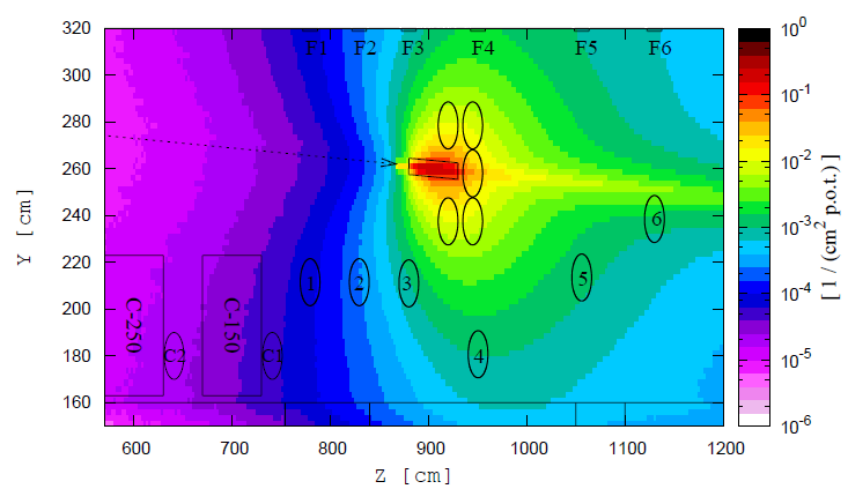

Fig. 2 High-energy hadron fluence normalized per primary particle of the $120 \mathrm{GeV} / \mathrm{c}$ mixed hadron beam particle $(60.7 \%$ $\pi+, 34.8 \% \mathrm{p}, 4.5 \% \mathrm{~K}+$ ) impinging on the copper target.

Table 1 Comparison between FLUKA simulation and RadMon measurements as performed during the CERF benchmark measurements. Results are expressed as high-energy hadron fluence per primary proton impinging on the CERF target. Stated errors are based on counts and calibration uncertainties for the RadMon values, as well as statistical uncertainties for the FLUKA calculations.

\begin{tabular}{cccc}
\hline Location & RadMon [Error] & FLUKA [Error] & $\begin{array}{c}\text { Ratio } \\
\text { (R/F) }\end{array}$ \\
\hline Pos1 & $3.77 \times 10^{-4}[20.0 \%]$ & $4.17 \times 10^{-4}[5.1 \%]$ & 0.90 \\
\hline Pos2 & $5.76 \times 10^{-4}[20.0 \%]$ & $5.76 \times 10^{-4}[4.6 \%]$ & 1.00 \\
\hline Pos3 & $1.99 \times 10^{-3}[20.0 \%]$ & $1.97 \times 10^{-3}[2.8 \%]$ & 1.04 \\
\hline Pos4 & $1.75 \times 10^{-3}[20.0 \%]$ & $1.71 \times 10^{-3}[3.4 \%]$ & 1.02 \\
\hline Pos5 & $1.53 \times 10^{-3}[20.0 \%]$ & $1.67 \times 10^{-3}[3.2 \%]$ & 0.92 \\
\hline Pos6 & $2.19 \times 10^{-3}[20.0 \%]$ & $2.19 \times 10^{-3}[2.9 \%]$ & 1.00 \\
\hline
\end{tabular}

During the CERF experiment these RadMons were replaced between the different measurement positions to study their response in the various mixed radiation field which are representative for the LHC. For the same setup FLUKA simulations were performed including all relevant details (geometry, materials, source term, etc...). For all measurement locations the total ionizing dose, $1 \mathrm{MeV}$ neutron equivalent and high-energy hadron fluence, as well as the particle energy spectra for the various types of particles were calculated. A horizontal cut through the layout and its respective high-energy hadron (> $20 \mathrm{MeV}$ ) radiation field can be seen in Fig. 2.

The measured data and the FLUKA calculations at the different measurement positions were compared with as shown in Table $\mathbf{1}$ and a very good agreement was obtained.

\section{The CNRAD Radiation Test Areas}

In 2007, electronics installed close to the CNGS ${ }^{17)}$ neutrino target area was suffering from Single Event Effect (SEE) errors in the equipment installed in the side gallery

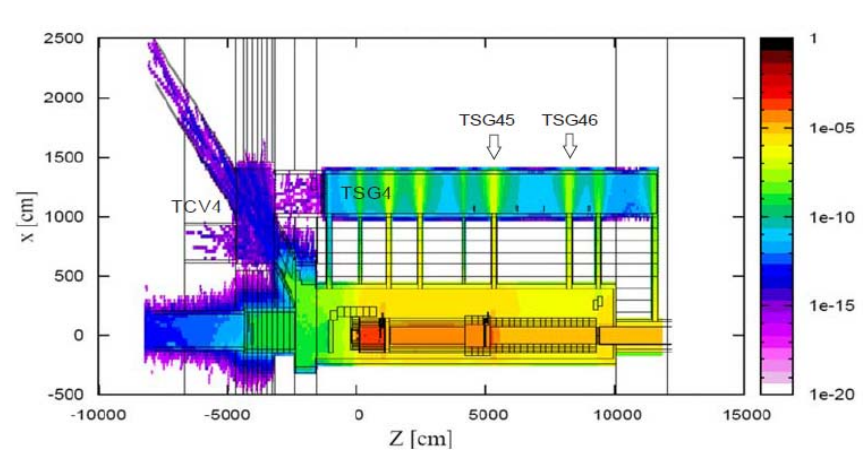

Fig. 3 FLUKA simulation of the high-energy hadron fluence $(\mathrm{E}>20 \mathrm{MeV})$ distribution in CNGS normalized to one primary $400 \mathrm{GeV} / \mathrm{c}$ proton on the graphite target. The CNRAD test areas are marked with arrows and the color code refers to number of particles per square-centimeter above $20 \mathrm{MeV}\left[\mathrm{cm}^{-2}\right]$.

Table 2 High-energy hadron fluence $\left(\mathrm{cm}^{-2}\right)$ for the two measurement positions (line-of-sight in transfer tunnel). Results are given as fluence per primary proton impinging on the CNGS target, as measured with the RadMon detector and compared to FLUKA simulation results. Stated errors are based on counts and calibration uncertainties for the RadMon values, as well as statistical uncertainties for the FLUKA calculations.

\begin{tabular}{cccc}
\hline Location & $\begin{array}{c}\text { RadMon } \\
\text { [Error] }\end{array}$ & $\begin{array}{c}\text { FLUKA } \\
\text { [Error] }\end{array}$ & $\begin{array}{c}\text { Ratio } \\
\text { (R/F) }\end{array}$ \\
\hline \multirow{2}{*}{ TSG45 } & $\begin{array}{c}1.9 \times 10^{-7} \\
{[20.0 \%]}\end{array}$ & $\begin{array}{c}2.1 \times 10^{-7} \\
{[5.7 \%]}\end{array}$ & 0.9 \\
\hline \multirow{2}{*}{ TSG46 } & $\begin{array}{c}2.0 \times 10^{-8} \\
{[20.0 \%]}\end{array}$ & $\begin{array}{c}1.9 \times 10^{-8} \\
{[6.8 \%]}\end{array}$ & 1.05 \\
\hline
\end{tabular}

(TSG4). The facility was suspended due to this problem, requiring that all the electronics was relocated to an adjacent area (ventilation chamber: TCV4) and shielded. The empty TSG4 gallery then became a testing area for the electronics systems installed in the LHC. Its acronym is CNRAD. ${ }^{18)}$

For the test area, the graphite target together with the horn and reflector located downstream are the source of the radiation. Secondary particles from the lateral shower propagate through the interconnecting galleries between the target chamber and the side gallery. The $400 \mathrm{GeV} / \mathrm{c}$ proton beam for CNGS is produced by several fast and high-intensity $\left(2.4 \times 10^{13}\right.$ protons) extractions from SPS. The test zones are concentrated around two adjacent ducts called TSG45 and TSG46 (see Fig. 3).

The dose rates in the test locations range from 0.2 to $30 \mathrm{~Gy} /$ week which corresponds to a respective range of $10^{8}-10^{10} \mathrm{~cm}^{-2} /$ week of high-energy hadrons and $1 \mathrm{MeV}$ neutron equivalent. The radiation fields are very stable throughout the year as the alignment of the beam has to be extremely precise. The integrated radiation levels thus scale with the integrated beam on target intensity and each test position can be calibrated just once, which is done with the dedicated monitors (RadMon) ${ }^{15,16)}$ and cross checked with activation foils and passive dosimeters, as well as successfully compared to detailed Monte Carlo calculations. 


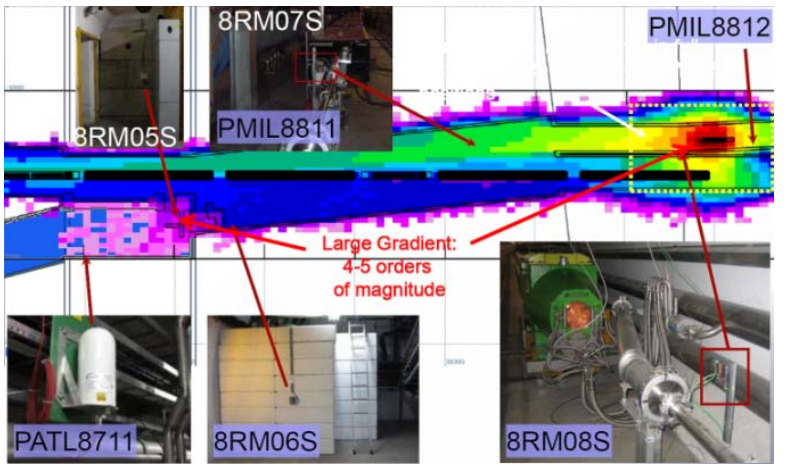

Fig. 4 Layout of the LHC injection line where it enters the LHC tunnel and as implemented in FLUKA. In addition, the various monitor locations are shown.

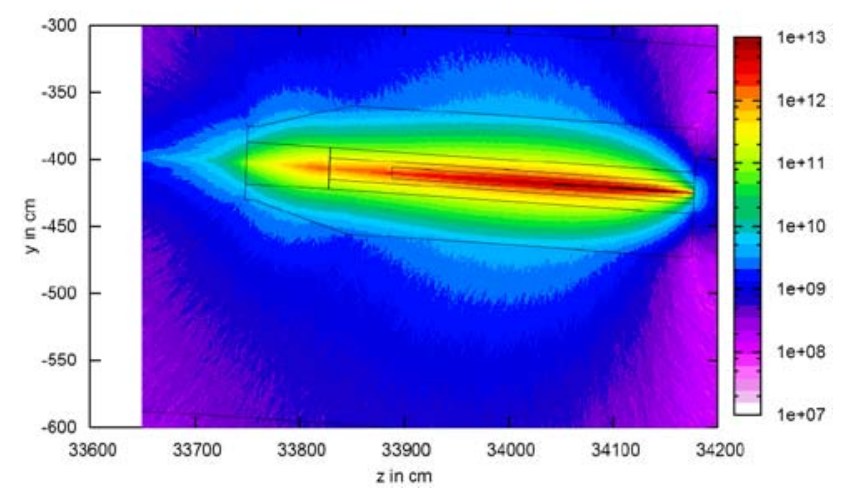

Fig. 5 Horizontal high-energy hadron fluence distribution around the TI8 beam stopper (TED) for $6.8 \times 10^{12} 450 \mathrm{GeV} / \mathrm{C}$ protons impinging on the TED. The color code refers to number of particles per square-centimeter above $20 \mathrm{MeV}\left[\mathrm{cm}^{-2}\right]$ and for the considered number of protons impinging on the TED.

A comparison for the radiation levels at the test locations between the FLUKA simulations and the measurements is shown in Table 2.

\section{LHC Application Benchmark during Injection Tests}

During LHC injection tests, monitor readings of RadMon detectors were analyzed and compared to respective FLUKA simulation results. The complex layout (see Fig. 4) of this injection section into the LHC (more than $100 \mathrm{~m}$ long) allowed for a detailed practical benchmark in order to verify the FLUKA predictions made for dedicated measurements.

During the first benchmark the RadMon was placed directly behind a beam absorber (TED) which is situated at the end of the LHC injection line. The TED can be moved into the beam in order to allow for setup and tuning studies to be carried out in the injection line without bringing beam into the LHC. This allows for an ideal benchmarking setup where the loss term (number of protons impinging on the TED) are known in all details. The high-energy hadron fluence distribution as well as the successful comparison between measurements and simulations are shown in Fig. 5 and in Table 3.

Beam losses occur not only on the TED (during injection line setup) but also during injection when fractions of the
Table 3 Comparison between FLUKA simulation and RadMon measurements performed during the injection test with direct dump on the TI8 beam stopper (TED). Stated errors are based on counts and calibration uncertainties for the RadMon values, as well as statistical uncertainties for the FLUKA calculations.

\begin{tabular}{cccc}
\hline Quantity & $\begin{array}{c}\text { RadMon } \\
\text { [Error] }\end{array}$ & $\begin{array}{c}\text { FLUKA } \\
\text { [Error] }\end{array}$ & $\begin{array}{c}\text { Ratio } \\
\text { (R/F) }\end{array}$ \\
\hline $\begin{array}{c}\text { High-energy } \\
\text { hadrons } \\
\left.\text { (cm }^{-2}\right)\end{array}$ & $\begin{array}{c}1.2 \times 10^{10} \\
{[20.0 \%]}\end{array}$ & $\begin{array}{c}0.96 \times 10^{10} \\
{[3.2 \%]}\end{array}$ & 0.80 \\
\hline $\begin{array}{c}\mathbf{1} \text { MeV neu- } \\
\text { tron equiv. } \\
\left.\text { (cm }^{-2}\right)\end{array}$ & $2 \times 10^{10}$ & $2.1 \times 10^{10}$ & 1.05 \\
\hline $\begin{array}{c}{[20.0 \%]} \\
\text { Dose } \\
\text { (Gy) }\end{array}$ & $\begin{array}{c}4.73 \\
{[20.0 \%]}\end{array}$ & $\begin{array}{c}5.0 \\
{[10 \%]}\end{array}$ & 1.06 \\
\hline
\end{tabular}

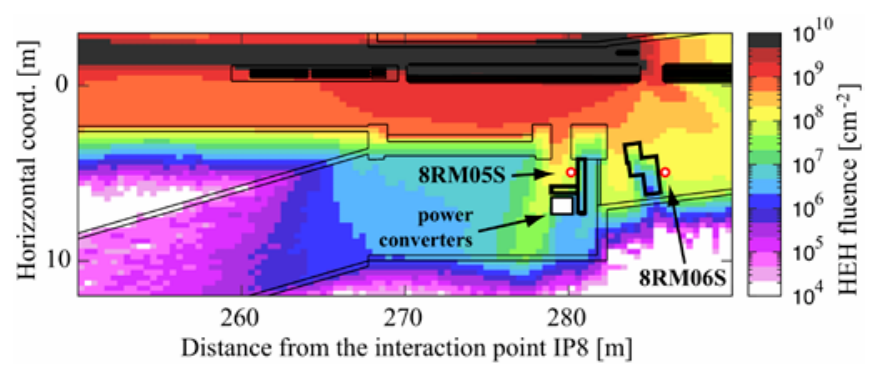

Fig. 6 High-energy hadron fluence distribution around the collimator (TCDI) location originating from losses of $450 \mathrm{GeV} / \mathrm{c}$ protons injected into the LHC. Fluences are normalized for an estimated cumulative annual loss on the respective collimator.

injected beam will be lost on the injection collimator (TCDI). Electronics near the shielded area has therefore to be sufficiently protected. Figure 6 shows the radiation field distribution in case of a beam loss on the TCDI, as well as the two monitor locations of interest.

During a setup of the collimator with a known number of protons impinging on its jaw, the two monitor locations (8RM05S and 8RM06S, see Fig. 4 and 6) then served as application benchmark in order to compare the FLUKA simulations and the detector readings. The good agreement is shown in Table 4, where the results are expressed as high-energy hadron fluence per primary particle interaction on the collimator jaw.

In addition to the injection tests numerous FLUKA calculations were required in the framework of the R2E study group for the LHC. Particular care was taken in a thorough understanding of the LHC radiation field, its impact on electronics, as well as the required Monte Carlo calculations and corresponding methods. ${ }^{19)}$ Continuous comparisons with multiple monitor readings form an integral part in the process of minimizing the risk of failures in electronic equipment induced by radiation originating from primary proton losses in the energy range of $450 \mathrm{GeV} / \mathrm{c}$ up to $7 \mathrm{TeV} / \mathrm{c}$. 
Table 4 High-energy hadron fluence $\left(\mathrm{cm}^{-2}\right)$ for two RadMon measurement positions close to the shielded area. Results are given as fluence per primary proton impinging on the injection collimator (TCDI). Stated errors are based on counts and calibration uncertainties for the RadMon values, as well as statistical uncertainties for the FLUKA calculations.

\begin{tabular}{cccc}
\hline Location & $\begin{array}{c}\text { RadMon } \\
\text { [Error] }\end{array}$ & $\begin{array}{c}\text { FLUKA } \\
\text { [Error] }\end{array}$ & $\begin{array}{c}\text { Ratio } \\
\text { (R/F) }\end{array}$ \\
\hline $\begin{array}{c}\text { 8RM05S } \\
\text { (3V) }\end{array}$ & $\begin{array}{c}6.97 \times 10^{-5} \\
{[20.0 \%]}\end{array}$ & $\begin{array}{c}7.75 \times 10^{-5} \\
{[5.7 \%]}\end{array}$ & 0.9 \\
\hline $\begin{array}{c}\text { 8RM06S } \\
\text { (5V) }\end{array}$ & $\begin{array}{c}6.36 \times 10^{-6} \\
{[20.0 \%]}\end{array}$ & $\begin{array}{c}6.10 \times 10^{-6} \\
{[6.8 \%]}\end{array}$ & 1.05 \\
\hline
\end{tabular}

\section{Conclusion}

The FLUKA code forms an integral part of all radiation damage to electronics studies for the LHC accelerator. In its integrality it is a very effective code and offers a comprehensive and efficient simulation environment through its available interfaces. It provides the simulation of proton and heavy ion collisions at LHC energies coupled with all scoring and biasing options important for electronics studies.

All physics models in FLUKA are individually benchmarked against experimental data and a wealth of benchmark studies for "real-life" applications exists. Furthermore, relatively little freedom in model selection by the user along with default settings for most of the free parameters allows a high level of quality assurance which leads to a stable application of the code and consistent results among different users.

In the context of radiation damage to electronics studies and the need of defining required mitigation actions around the LHC accelerator, FLUKA calculations allow for a reliable characterization of the radiation field, define the requirements for radiation tolerant electronics design and test requirements, as well as shielding developments or, if needed, relocation campaigns.

Benchmark experiments at dedicated test facilities, as well as early LHC operation demonstrate the very good agreement between FLUKA predictions and measurement results. The selection of application benchmark measurements presented here and the large field of related CERN applications nicely demonstrates the FLUKA capabilities for applications related to radiation damage to electronics. This can range from the study of device effects, the detailed characterization of the radiation field and radiation monitor calibration, to the input requirements for important mitigation studies including shielding, relocation or other options.

\section{Acknowledgment}

The authors are grateful to numerous discussions between the various members of the R2E Study Group, as well as the CERN Radiation Working Group.

\section{References}

1) A.Ferrari, P.Sala, A.Fassò, J. Ranft, FLUKA: A Multi-Particle
Transport Code, CERN-2005-10 (2005), INFN/TC_05/11, SLAC-R-773.

2) G. Battistoni, S. Muraro, P. R. Sala, F. Cerutti, A. Ferrari, S. Roesler, A. Fassò, J. Ranft, "The FLUKA code: Description and benchmarking," Proc. the Hadronic Shower Simulation Workshop 2006, Fermilab 6-8 September 2006, M. Albrow, R. Raja (eds.), AIP Conference Proceeding 896, 31-49 (2007).

3) A. Holmes-Siedle, L. Adams, Handbook of Radiation Effects, Oxford University Press, ISBN 978-0-19-850733-8, (2007).

4) CERN European Organisation for Nuclear Research, www.cern.ch

5) O. Brüning, P. Collier, P. Lebrun, S. Myers, R. Ostojic, J. Poole, P. Proudlock, LHC Design Report v.1: the LHC Main Ring, CERN-2004-003-V-1, ISBN 9789290832249, (2004).

6) M. Huhtinen, P. A. Aarnio, "Pion induced displacement damage in silicon devices," Nucl. Instr. Meth. Phys. Res., A335, 580-582 (1993).

7) G. P. Summers et al., "Damage correlations in semiconductors exposed to gamma, electron and proton radiations," IEEE Trans. Nucl. Sci., 40, No. 6 (1993).

8) V. Vlachoudis et al., "FLUKA Realistic Modeling of Radiation Induced Damage," Prog. Nucl. Sci. Technol., this volume, (2010).

9) D. Kramer et al., "LHC RadMon SRAM detectors used at different voltages to determine the thermal neutron to high-energy hadron fluence ratio," RADECS conference 2010, to be published, (2010).

10) M. Brugger et al., "Review of critical radiation areas for LHC Electronics and mitigation actions. Radiation monitoring and first results," Radiation-To-Electronic Study Group (R2E), CERN LHC Performance Workshop, Chamonix (2010).

11) H. Nakashima et al., "Current Status of the AGS Spallation Target Experiment," Proc. OECD/NEA Workshop on Shielding Aspects on Accelerator, Target and Irradiation Facilities-SATIF6, SLAC, 10-12 Apr. 2002, pp.27-36 (2002).

12) H. Hirayama et al., "Inter-comparison of medium-energy neutron attenuation in iron and concrete," Proc. the 10th Meeting of the Task Force on Shielding Aspects of Accelerators, Targets and Irradiation Facilities, SATIF-10, CERN 2-4 June 2010, to be published, (2010).

13) S. Roesler et al., "The Application of the Monte Carlo Code FLUKA in Radiation Protection Studies for the Large Hadron Collider,” Prog. Nucl. Sci. Technol., this volume, (2010).

14) A. Mitaroff, M. Silari, "The CERN-EU high-energy Reference Field (CERF) facility for dosimetry at commercial flight altitudes and in space,” Radiat. Prot. Dosim., 102, 7-22 (2002).

15) T. Wijnands, C. Pignard, R. Tesarek., "An on line radiation monitoring system for the LHC machine and experimental caverns," Proc. 12th Workshop on Electronics for LHC and Future Experiments (2006).

16) D. Kramer et al., "LHC RadMon SRAM detectors used at different voltages to determine the thermal neutron to high energy hadron fluence ratio," IEEE Trans. Nucl. Sci., jn press.

17) E. Gschwendtner et al., The CNGS Facility: Performance And Operational Experience, EuCARDCON-2009-014 (2009).

18) D. Kramer et al., "CERN CNRAD test area for LHC electronics, Summary of the 2009 irradiation tests and perspectives for future tests and facilities”, CERN LHC Performance Workshop, Chamonix (2010).

K. Roeed et al., "FLUKA simulations for SEE Studies of Critical LHC Underground Areas”, RADECS conference 2010, to be published, (2010). 